\title{
577.
}

\section{NOTE IN ILLUSTRATION OF CERTAIN GENERAL THEOREMS OBTAINED BY DR LIPSCHITZ.}

[From the Quarterly Journal of Pure and Applied Mathematics, vol. XII. (1873), pp. $346-349$.]

THE paper by Dr Lipschitz, which follows the present Note [in the Quarterly Journal, l.c.], is supplemental to Memoirs by him in Crelle, vols. LXx., LxxII., and Lxxiv.; and he makes use of certain theorems obtained by him in these memoirs; these theorems may be illustrated by the consideration of a particular example.

Imagine a particle not acted on by any forces, moving in a given surface; and let its position on the surface at the time $t$ be determined by means of the general coordinates $x, y$. We have then the vis-viva function $T$, a given function of $x, y, x^{\prime}, y^{\prime}$; and the equations of motion are

$$
\frac{d}{d t} \frac{d T}{d x^{\prime}}-\frac{d T}{d x}=0, \quad \frac{d}{d t} \frac{d T}{d y^{\prime}}-\frac{d T}{d y}=0,
$$

which equations sorve to determine $x, y$ in terms of $t$, and of four arbitrary constants; these are taken to be the initial values (or values corresponding to the time $t=t_{0}$ ) of $x, y, x^{\prime}, y^{\prime}$; say the values are $\alpha, \beta, \alpha^{\prime}, \beta^{\prime}$.

We have the theorem that $x, y$ are functions of $\alpha, \beta, \alpha^{\prime}\left(t-t_{0}\right), \beta^{\prime}\left(t-t_{0}\right)$.

Suppose for example that $x, y, z$ denote ordinary rectangular coordinates, and that the particle moves on the sphere $x^{2}+y^{2}+z^{2}=c^{2}$; to fix the ideas, suppose that the coordinates $z$ are measured vertically upwards, and that the particle is on the upper hemisphere; that is, take $z=+\sqrt{ }\left(c^{2}-x^{2}-y^{2}\right)$, we have

$$
T=\frac{1}{2}\left(x^{\prime 2}+y^{\prime 2}+z^{\prime 2}\right) \text {, }
$$

where $z^{\prime}$ denotes its value in terms of $x, y, x^{\prime}, y^{\prime}$; viz. we have $x x^{\prime}+y y^{\prime}+z z^{\prime}=0$, or

$$
z^{\prime}=-\frac{x x^{\prime}+y y^{\prime}}{z},=-\frac{x x^{\prime}+y y^{\prime}}{\sqrt{ }\left(c^{2}-x^{2}-y^{2}\right)}
$$


the proper value of $T$ is thus

$$
=\frac{1}{2}\left\{x^{\prime 2}+y^{\prime 2}+\frac{\left(x x^{\prime}+y y^{\prime}\right)^{2}}{c^{2}-x^{2}-y^{2}}\right\}, \quad .
$$

but it is convenient to retain $z, z^{\prime}$, taking these to signify throughout their foregoing values in terms of $x, y, x^{\prime}, y^{\prime}$.

The constants of integration are, as before, $\alpha, \beta, x^{\prime}, \beta^{\prime}$; but we use also $\gamma, \gamma^{\prime}$ considered as signifying given functions of these constants, viz. we have

$$
\gamma=\sqrt{ }\left(c^{2}-\alpha^{2}-\beta^{2}\right) \text { and } \gamma^{\prime}=-\frac{\alpha \alpha^{\prime}+\beta \beta^{\prime}}{\sqrt{ }\left(c^{2}-\alpha^{2}-\beta^{2}\right)},
$$

(in fact, $\alpha^{2}+\beta^{2}+\gamma^{2}=c^{2}$ and $\alpha \alpha^{\prime}+\beta \beta^{\prime}+\gamma \gamma^{\prime}=0 ; \gamma, \gamma^{\prime}$ being thus the initial values of $\left.z, z^{\prime}\right)$.

Now, writing

$$
\sigma=\frac{\left(t-t_{0}\right) \sqrt{ }\left(\alpha^{\prime 2}+\beta^{\prime 2}+\gamma^{\prime 2}\right)}{c},
$$

the required values of $x, y$ and the corresponding value of $z$ are

$$
\begin{aligned}
& x=\alpha \cos \sigma+\frac{c \alpha^{\prime}}{\sqrt{\left(\alpha^{\prime 2}+\beta^{\prime 2}+\gamma^{\prime 2}\right)}} \sin \sigma, \\
& y=\beta \cos \sigma+\frac{c \beta^{\prime}}{\sqrt{\left(\alpha^{\prime 2}+\beta^{\prime 2}+\gamma^{\prime 2}\right)}} \sin \sigma, \\
& z=\gamma \cos \sigma+\frac{c \gamma^{\prime}}{\sqrt{\left(\alpha^{\prime 2}+\beta^{\prime 2}+\gamma^{\prime 2}\right)}} \sin \sigma .
\end{aligned}
$$

To verify that these are functions of $\alpha, \beta, \alpha^{\prime}\left(t-t_{0}\right), \beta^{\prime}\left(t-t_{0}\right)$, write $\alpha^{\prime}\left(t-t_{0}\right)=u$, $\beta^{\prime}\left(t-t_{0}\right)=v$; and take also $\gamma^{\prime}\left(t-t_{0}\right)=w$; we have $\alpha u+\beta v+\gamma w=0$, viz. $w,=-\frac{1}{\gamma}(\alpha u+\beta v)$, is a function of $\alpha, \beta, u, v$; and then

and

$$
\sigma=\frac{\sqrt{ }\left(u^{2}+v^{2}+w^{2}\right)}{c}
$$

$$
\begin{aligned}
& x=\alpha \cos \sigma+\frac{u}{\sigma} \sin \sigma, \\
& y=\beta \cos \sigma+\frac{v}{\sigma} \sin \sigma \\
& z=\gamma \cos \sigma+\frac{w}{\sigma} \sin \sigma
\end{aligned}
$$

so that $x, y$ (and also $z$ ) are each of them a function of $\alpha, \beta, u, v$, that is $\alpha, \beta, \alpha^{\prime}\left(t-t_{0}\right)$, $\beta^{\prime}\left(t-t_{0}\right)$, which is the theorem in question.

The original variables are $x, y$; the quantities $\alpha^{\prime}\left(t-t_{0}\right), \beta^{\prime}\left(t-t_{0}\right)$, or $u, v$ are Dr Lipschitz' "Normal-Variables," and the theorem is that the original variables are functions of their initial values, and of the normal-variables. 
The vis-viva function $T$ may be expressed in terms of the normal-variables and their derived functions; viz, it is easy to verify that we have

$$
\begin{gathered}
T=\frac{1}{2}\left(\frac{1}{c^{2} \sigma^{2}}-\frac{\sin ^{2} \sigma}{c^{2} \sigma^{4}}\right)\left(u u^{\prime}+v v^{\prime}+w w^{\prime}\right)^{2} \\
-\frac{1}{2} \frac{\sin ^{2} \sigma}{\sigma}\left(u^{\prime 2}+v^{\prime 2}+w^{\prime 2}\right),
\end{gathered}
$$

where $w$ denotes $-\frac{1}{\gamma}(\alpha u+\beta v)$ and consequently $w^{\prime}$ denotes $-\frac{1}{\gamma}\left(\alpha u^{\prime}+\beta v^{\prime}\right)$; introducing herein differentials instead of derived functions, or writing

$$
\begin{aligned}
\phi(d u) & =\frac{1}{2}\left(\frac{1}{c^{2} \sigma^{2}}-\frac{\sin ^{2} \sigma}{c^{2} \sigma^{4}}\right)(u d u+v d v+w d w)^{2} \\
& +\frac{1}{2} \frac{\sin ^{2} \sigma}{\sigma^{2}}\left(d u^{2}+d v^{2}+d w^{2}\right),
\end{aligned}
$$

where $w, d w$ denote $-\frac{1}{\gamma}(\alpha u+\beta v),-\frac{1}{\gamma}(\alpha d u+\beta d v)$ respectively; then $\phi(d u)$ is the function thus denoted by Dr Lipschitz: and writing herein $t-t_{0}=0$, and thence $u=0$, $v=0, w=0, \sigma=0$, the resulting value of $\phi(d u)$ is

$$
f_{0}(d u),=\frac{1}{2}\left(d u^{2}+d v^{2}+d w^{2}\right)
$$

where $f_{0}(d u)$ is the function thus denoted by him; the corresponding value of $f_{0}(u)$ is $=\frac{1}{2}\left(u^{2}+v^{2}+w^{2}\right)$. We have thus an illustration of his theorem that the function $\phi(d u)$ is such that we have identically

$$
\phi(d u)-\left\{d \sqrt{ }\left\{f_{0}(u)\right\}\right\}^{2}=\frac{m^{2}}{2 f_{0}(u)}\left[f_{0}(d u)-\left\{d \sqrt{ }\left(f_{0} u\right)\right\}^{2}\right],
$$

where $m$ is a function of $u, v$ independent of the differentials $d u, d v$; the value in the present example is in fact $m^{2}=c^{2} \sin ^{2} \sigma$; or the identity is

$$
\phi(d u)-\left\{d \sqrt{ }\left(f_{0} u\right)\right\}^{2}=\frac{c^{2} \sin ^{2} \sigma}{2\left(f_{0} u\right)}\left[f_{0}(d u)-\left\{d \sqrt{ }\left(f_{0} u\right)\right\}^{2}\right],
$$

in verification whereof observe that we have

$$
\begin{aligned}
d \sqrt{ }\left(f_{0} u\right)=\frac{d f_{0}(u)}{2 \sqrt{ }\left(f_{0} u\right)} & =\frac{u d u+v d v+w d w}{\sqrt{ }\left(u^{2}+v^{2}+w^{2}\right)} \\
& =\frac{1}{c \sigma}(u d u+v d v+w d w)^{2} .
\end{aligned}
$$

The value of the left-hand side is thus

viz. this is

$$
=-\frac{\sin ^{2} \sigma}{c^{2} \sigma^{4}}(u d u+v d v+w d w)^{2}+\frac{1}{2} \frac{\sin ^{2} \sigma}{\sigma^{2}}\left(d u^{2}+d v^{2}+d w^{2}\right)
$$

or, finally, it is

$$
=\frac{c^{2} \sin ^{2} \sigma}{c^{2} \sigma^{2}}\left\{\frac{1}{2}\left(d u^{2}+d v^{2}+d w^{2}\right)-\frac{1}{c^{2} \sigma^{2}}(u d u+v d v+w d w)^{2}\right\}
$$

which is right.

$$
=\frac{c^{2} \sigma^{2}}{2 f_{0}(u)}\left\{f_{0}(d u)-\left[d \sqrt{ }\left\{f_{0}(u)\right\}\right]^{2}\right\},
$$

